\title{
AVALIAÇÃO ECONÔMICA DO CULTIVO DE TRIGO DOS ESTADOS DO RIO GRANDE DO SUL E PARANÁ
}

\author{
Matheus Wemerson Gomes Pereira \\ Alan Figueiredo de Arêdes ${ }^{2}$ \\ Erly Cardoso Teixeira ${ }^{3}$
}

Resumo - O objetivo deste artigo foi analisar a viabilidade econômica do cultivo de trigo nos Estados do Rio Grande do Sul (RS) e Paraná (PR), considerando ou não as condições de risco. A metodologia aplicada inclui os indicadores de viabilidade econômica Benefício-Custo, Valor Presente Líquido, Taxa Interna de Retorno, Taxa Interna de Retorno Modificada e utilização do método de Monte Carlo. Os resultados mostram que a produção tritícola é economicamente viável, especialmente no Estado do Paraná.

Palavras-chave: Rio Grande do Sul, Paraná, trigo, viabilidade econômica.

\section{Introdução}

Na safra 2007/08, o Brasil produziu cerca de 3.832 toneladas de trigo, um crescimento de 71,50\% em relação à safra 2006/07, o que se deve, principalmente, à elevação da produtividade em $65,70 \%$, enquanto a área para cultivo cresceu apenas 3,50\% (CONAB, 2008).

O trigo é uma cultura de inverno, e sua produção concentra-se, principalmente na região do Sul do país, especialmente nos Estados do Paraná e Rio Grande do Sul, que apresentam clima ameno e temperaturas baixas. Na safra 2007/08, esses estados produziram um total de $1.853 \mathrm{e}$ 1.528 toneladas, respectivamente, ou seja, juntos somaram $88,26 \%$ da produção nacional do grão (CONAB, 2008), o que não deixa dúvidas da importância desses estados no abastecimento de trigo no país.

\footnotetext{
Economista; doutorando em Economia Aplicada, DER/UFV, bolsista do CNPq. E-mail: matheuswgp@yahoo.com.br

2 Economista; doutorando em Economia Aplicada, DER/UFV, bolsista do CNPq. E-mail: aredess@ yahoo.com.br

3 Ph.D.; Professor Titular; Departamento de Economia Rural, UFV.E-mail: Teixeira@ufv.br
} 
É uma cultura que pode ser utilizada na rotatividade da terra para controle de doenças, como o nematóide de cisto da soja (Heterodera glycines Ichinohe), que é a principal doença responsável por perdas na produção de culturas como soja, feijão e ervilha. De acordo com a Embrapa (2007), a utilização de cultivares resistentes associadas à rotação com culturas não-hospedeiras, entre elas, o trigo, é o método mais econômico e eficiente para manter o controle do nematóide.

A produção de commodities agrícolas, entre elas o trigo, depende de fatores considerados instáveis, como os ambientais, oferta e demanda interna e externa, e, ainda, de fatores de comercialização, que exercem grande influência na variabilidade da produção e dos preços, impondo dificuldades no planejamento da produção e no abastecimento de mercados.

Dessa forma, na atividade agrícola devem-se considerar tanto o nível de rentabilidade das culturas quanto os seus riscos. Entre os riscos que afetam a agricultura, os mais comuns são os de mercado e de produção. O primeiro é responsável pela oscilação do nível de preço e o segundo, pela oscilação da produtividade.

O presente artigo teve por objetivou quantificar o retorno e os riscos no cultivo de trigo nos Estados do Rio Grande do Sul e Paraná, com vistas em fornecer ao produtor medidas de retornos e riscos inerentes à atividade. Para isso, foram elaborados os fluxos de caixa e obtidos os indicadores de viabilidade econômica, considerando ou não condições de risco em ambos os estados. 


\section{Metodologia}

\section{1. Análise de projetos de investimento}

Dentre as etapas de elaboração de projetos, destacam-se as análises quantitativas e qualitativas, que resultarão no fluxo de caixa do projeto. A correta elaboração do fluxo de caixa é de suma importância, uma vez que os indicadores de rentabilidade e risco do projeto são derivados dele, ou seja, das entradas e saídas de numerários ocorridas durante o período de sua vigência.

Entre os indicadores econômicos de viabilidade de projetos, foram utilizados Benefício-Custo (B/C), Valor Presente Líquido (VPL), Taxa Interna de Retorno (TIR) e Taxa Interna de Retorno Modificada (TIRM) ${ }^{4}$.

Razão Benefício-Custo (B/C): é a razão entre receitas e despesas que permite obter o retorno do investimento por unidade monetária investida, descontado o valor do dinheiro no tempo, a uma taxa de desconto predeterminada. Em forma de equação:

$$
B / C=\sum_{t=0}^{n} \frac{B_{t} /(1+r)^{t}}{C_{t} /(1+r)^{t}},
$$

em que B são as receitas; $\mathrm{C}$, custos variáveis e investimentos; $\mathrm{t}$, período de tempo; n, tempo-limite; e r, a taxa de desconto adotada. Quando B/ C>1, o projeto será economicamente viável.

Valor Presente Líquido (VPL): representa o retorno monetário do investimento considerando o valor do dinheiro no tempo, a uma taxa de desconto predeterminada. Quando VPL $>0$, o projeto será economicamente viável. Em forma de equação:

Para mais detalhes, ver Buarque (1991), Woiler e Mathias (1996), Ross et. al. (1998), Rezende e Oliveira (2001). 
$V P L=\sum_{t=0}^{n}(B-C)_{t} /(1+r)^{t}$

em que B são os benefícios; $\mathrm{C}$, custos e valores dos investimentos gerados pelo projeto; $\mathrm{t}$, período de tempo; $\mathrm{n}$, tempo-limite; e r, taxa de desconto predeterminada.

Taxa Interna de Retorno (TIR): é a taxa de desconto interna gerada pelo projeto que torna o VPL $=0$. Em forma de equação:

$$
V P L=\sum_{t=0}^{n}(B-C)_{t} /\left(1+r^{*}\right)^{t}=0
$$

em que B, C, t e n são definidos como anteriormente; e r* é a taxa de desconto interna (TIR). Quando a TIR for maior que a taxa de desconto predeterminada, o projeto será economicamente viável.

Taxa Interna de Retorno Modificada (TIRM): é um indicador que considera o custo de capital e os juros de reinvestimento dos fluxos de caixa. Em forma de equação:

$$
\text { TIRM }=\left[\frac{\sum_{t=0}^{n} B_{t}\left(1+r_{r}\right)^{n-t}}{\sum_{t=0}^{n} C_{t} /\left(1+r_{c}\right)^{t}}\right]^{\frac{1}{n}}-1
$$

em que B, C, t e n são definidos como anteriormente, sendo $r_{r}$ a taxa de reinvestimento e $r_{c}$ a taxa de juros correspondente ao custo de capital ou financiamento, os quais foram de 6\% ao ano. Quando a TIRM for maior 
que a taxa de desconto predeterminada, o projeto será economicamente viável.

\subsection{Análise de risco}

Além das análises de retorno, decisões acerca dos investimentos são influenciadas por outros fatores relativos ao ambiente de implantação do projeto, entre eles, a impossibilidade de prever as condições climáticas econômicas e locais que o envolvem. Dessa forma, na tomada de decisões relativas a investimentos considera-se determinado grau de incerteza e risco.

Segundo Woiler e Mathias (1996), o risco é algo inerente à própria vida do projeto, ou seja, é impossível eliminá-lo das ações a serem implementadas, devido à impossibilidade de coletar todas as informações relevantes ao projeto.

Considerar-se-á existência de risco quando forem conhecidos os possíveis estados futuros das principais variáveis que afetarem o projeto e suas respectivas probabilidades de ocorrência. Quando não forem identificados os possíveis comportamentos dessas variáveis, dir-se-á que há incerteza (Woiler; Mathias, 1996).

Gitman (1997) afirmam que o risco pode ser mensurado pelo desviopadrão, que mede a dispersão dos retornos em relação ao seu valor esperado ou médio. Como pode ser visto na Figura 1, em um mesmo nível de retorno médio, o desvio-padrão do projeto $\mathrm{B}$ será maior que o de $\mathrm{A}$, ou seja, o projeto $\mathrm{B}$ apresentará dispersão em torno da média bem superior à do projeto $\mathrm{A}$. Assim, o projeto $\mathrm{B}$ terá maior risco, uma vez que seu retorno terá maior variabilidade. 


\section{Probabilidade}

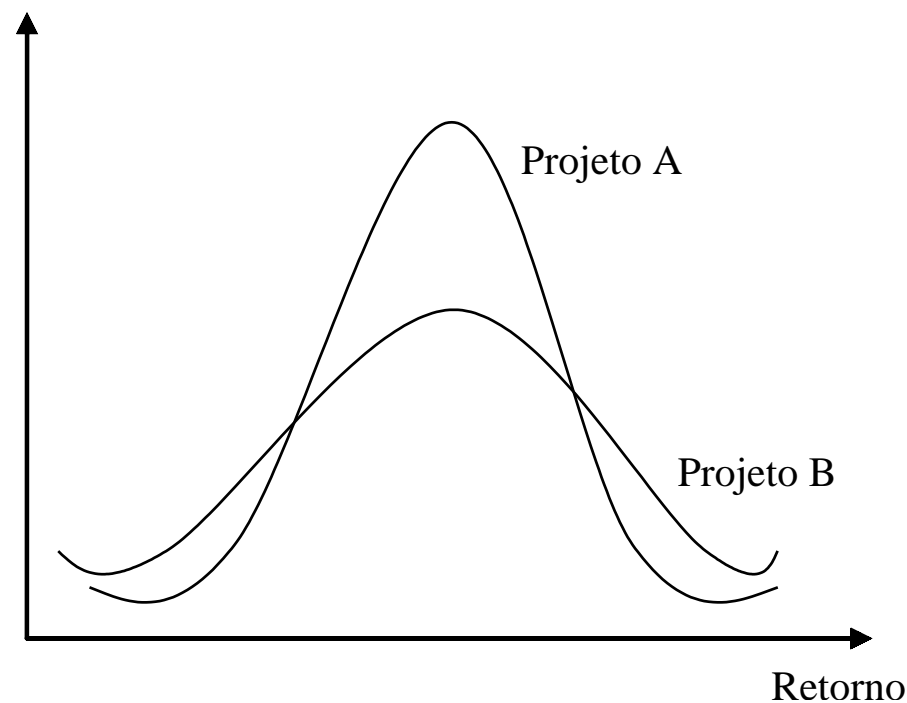

Figura 1 - Distribuição de probabilidades contínuas do retorno do ativo. Fonte: Gitman (1997).

O cálculo do desvio-padrão é feito pela seguinte equação:

$$
\sigma_{K}=\sqrt{\frac{\sum_{i=1}^{n}\left(K_{i}-\bar{K}\right)^{2}}{n-1}},
$$

em que $\sigma_{K}$ é o desvio-padrão dos retornos do ativo; $K$, retornos para cada observação $i$; $n$; número de observações analisadas; e $\bar{K}$, retorno médio, dado pela equação: 


$$
\bar{K}=\sum_{i=1}^{n} K_{i} / n
$$

De acordo com Gitman (1997), quando se quer medir e comparar riscos de projetos, ou ativos com diferentes retornos, usa-se o indicador de coeficiente de variação, pois é o mais indicado na análise da dispersão relativa dos retornos quando suas médias forem diferentes. $\mathrm{O}$ coeficiente de variação é determinado pela equação:

$$
C V=\sigma_{k} / \bar{K}
$$

em que CV é o coeficiente de variação. Quanto maior o CV, maior será o risco do ativo, visto que maior será a proporção do desvio-padrão em relação à média do retorno do ativo.

No entanto, essas medidas de risco são limitadas. Buarque (1991) e Noronha (1988) sugeriram uma melhor forma de medida do risco, dada pelos seguintes passos:

I. Identificação das principais variáveis que interferem nos retornos do investimento;

II. Cálculo dos possíveis valores para essas variáveis e suas probabilidades de realização;

III. Simulação em conjunto dos possíveis valores das variáveis, de acordo com suas probabilidades;

IV. Apresentação dos retornos obtida em forma de distribuição de probabilidade simples ou acumulada;

Essa análise de risco é aperfeiçoada pelo emprego do método de Monte Carlo, com base na utilização de funções de probabilidade e simulações aleatórias dos valores das variáveis que interferem no fluxo de caixa, o 
que possibilita obter, de forma probabilística, os valores dos indicadores econômicos.

Outro método de avaliação do risco, muito usado, é o de sensibilidade, em que se mede a sensibilidade do choque isolado de uma variável de entrada do fluxo de caixa, como preço e produtividade, bem como os efeitos do choque sobre a rentabilidade, por meio de um indicador econômico.

\subsection{Tratamento e fonte de dados}

O preço médio do trigo considerado foi de $\mathrm{R} \$ 26,54 / \mathrm{saca}$ de $60 \mathrm{~kg}$ e produtividade média de 45 sacas de $60 \mathrm{~kg} / \mathrm{ha}$, no Estado do Paraná, e de $\mathrm{R} \$ 25,11 / \mathrm{saca}$ de $60 \mathrm{~kg}$ e produtividade média de 40 sacas de $60 \mathrm{~kg} / \mathrm{há}$, no Rio Grande do Sul. A taxa de desconto ou de juros, utilizada nas análises como representação do valor do dinheiro ao longo do tempo, é de $0,59 \%$ ao mês, referente à taxa de juro real da caderneta de poupança em fevereiro de 2008. O horizonte temporal do investimento considerado foi de quatro meses, que consiste no intervalo entre o cultivo do trigo e a sua colheita.

$\mathrm{Na}$ análise de risco empregou-se o método de Monte Carlo, utilizando-se o software @Risk, tendo sido promovidas 15.000 interações aleatórias entre preços do grão; da produtividade; dos custos com colheita mecânica, uréia, fertilizante, sementes e fungicida; e taxa de juros, utilizando-se a distribuição de probabilidade normal para os preços e a triangular, para as demais variáveis.

Os dados referentes a custos de produção e preço dos grãos foram obtidos no Agrianual (2008). Os níveis de preço, produtividade e fluxos de caixa, elaborados para o investimento na produção do trigo nos Estados do Rio Grande do Sul e Paraná, são apresentados nas Tabelas 1, 2 e 3 (Anexos). 


\section{Resultados e discussão}

\subsection{Análise sem risco}

Pela utilização dos níveis de preço, produtividade e fluxos de caixa elaborados obtiveram-se os indicadores de avaliação econômica Benefício-Custo (B/C), Valor Presente líquido (VPL), Taxa Interna de Retorno (TIR) e Taxa Interna de Retorno Modificada (TIRM), que são apresentados na Tabela 4.

O indicador B/C, obtido por ha de trigo no Rio Grande do Sul, foi de 1,05, o que indica que os benefícios suplantaram os custos em $\mathrm{R} \$ 0,05$, isto é., cada $\mathrm{R} \$ 1,00$ aplicado na forma de investimento retornam apenas $\mathrm{R} \$$ 1,05 como benefício. Já no Estado do Paraná, o indicador B/C foi de 1,15. Assim, em ambos os Estados, o critério de decisão é que o investimento na produção do trigo é economicamente viável, visto que os benéficos econômicos foram superiores aos custos de investimento, à taxa de desconto de $0,59 \%$ ao mês (Tabela 4 ).

Pelo indicador VPL obtido, a triticultura gera um retorno presente líquido positivo, descontado o valor do dinheiro no tempo, à taxa de $0,59 \%$ ao mês. Dessa forma, remunera o capital investido e incrementa o valor de mercado do triticultor em R \$39,84/ha, no Rio Grande do Sul, e em R \$ 142,83/ha, no Paraná (Tabela 4). 
Tabela 4 - Indicadores de viabilidade econômica no cultivo de 1 ha de trigo nos Estados do Rio Grande do Sul e Paraná

\begin{tabular}{cccc}
\hline Indicador & Unidade & RS & PR \\
\hline B/C & - & 1,05 & 1,15 \\
VPL & $\mathrm{R} \$$ & 39,84 & 142,83 \\
TIR & $\%$ & 2,90 & 7,86 \\
TIRM & $\%$ & 2,62 & 7,00 \\
\hline
\end{tabular}

Fonte: Dados da pesquisa.

De acordo com o indicador TIR, a produção, em ambas as regiões, também é economicamente viável e gera uma taxa de retorno do capital investido de 2,90\% ao mês por ha, no Rio Grande do Sul, e de 7,86\% ao mês por ha, no Paraná, as quais são superiores à taxa de desconto de $0,59 \%$ ao mês. Na TIRM, ajustada pela taxa de desconto, os retornos do investimento foram de 2,62\% ao mês por ha no Rio Grande do Sul e de $7,00 \%$ ao mês por ha, no Paraná (Tabela 4), o que evidencia um maior retorno econômico da cultura no Paraná, que se deve, principalmente, ao fato de o preço e de a produtividade do trigo serem maiores nesse Estado, como pode ser visto nas Tabelas 1, 2 e 3 (Anexos).

\subsection{Análise com risco}

De acordo com a análise de sensibilidade, as variáveis preço e produtividade foram os maiores determinantes do retorno do capital investido, medido pelo Benefício-Custo $(\mathrm{B} / \mathrm{C})$, e não houve diferença significativa entre os efeitos dessas duas variáveis sobre a lucratividade do cultivo do grão.

Ao analisar a Tabela 5, constata-se que uma elevação de $1 \%$ no preço provocou aumento de 0,736 \% no B/C do Rio Grande do Sul e de 0,708\% no do Paraná. Já a elevação de $1 \%$ na produtividade promoveu elevação de $0,674 \%$ no B/C do Rio Grande do Sul e de $0,704 \%$ no do Paraná. Por 
outro lado, elevações nos níveis das demais variáveis tiveram efeitos negativos sobre o indicador $\mathrm{B} / \mathrm{C}$, uma vez que essas são variáveis de custos e não de receita, como o preço do trigo e sua produtividade (Tabela $5)$.

Tabela 5 - Análise de sensibilidade no cultivo de trigo nos Estados do Rio Grande do Sul e Paraná, em relação ao B/C

\begin{tabular}{cccc}
\hline Indicador & Unidade & RS & PR \\
\hline Preço & $\%$ & 0,736 & 0,708 \\
Produtividade & $\%$ & 0,674 & 0,704 \\
Fertilizante & $\%$ & $-0,041$ & $-0,038$ \\
Colheita mecânica & $\%$ & $-0,031$ & $-0,036$ \\
Fungicida & $\%$ & $-0,026$ & $-0,027$ \\
Uréia & $\%$ & $-0,015$ & $-0,020$ \\
Taxa de juros & $\%$ & $-0,001$ & $-0,001$ \\
\hline
\end{tabular}

Fonte: Dados da pesquisa.

Pela análise da viabilidade econômica sujeita ao risco no indicador B/C, no Estado do Rio Grande do Sul encontrou-se um B/C médio de 0,97 e um coeficiente de variação de $24,94 \%$. Já no Estado do Paraná, o B/C médio foi de 1,06 e o coeficiente de variação, de $23,81 \%$, o que indica que o cultivo do trigo no Paraná, além de gerar maior Benefício-Custo em termos médios, está sujeito ainda ao menor nível de risco, uma vez que seu coeficiente de variação foi menor, ou seja, a variabilidade (desviopadrão) do B/C, em relação à sua média, foi menor nesse estado (Tabela $6)$. 
Tabela 6 - Análise de risco no cultivo de 1 ha de trigo nos Estados do Rio Grande do Sul e Paraná, em relação ao B/C

\begin{tabular}{cccc}
\hline \multirow{2}{*}{ Descrição } & \multirow{2}{*}{ Unidade } & \multicolumn{2}{c}{ Estado } \\
\cline { 3 - 4 } & & RS & PR \\
\hline Mínimo & - & 0,10 & 0,31 \\
Máximo & - & 2,00 & 2,15 \\
Média & - & 0,97 & 1,06 \\
Desvio-padrão & - & 0,24 & 0,25 \\
Coeficiente de variação & $\%$ & 24,94 & 23,81 \\
Probabilidade B/C<1 & $\%$ & 45,00 & 40,00 \\
Probabilidade B/C $>1$ & $\%$ & 55,00 & 60,00 \\
\hline
\end{tabular}

Fonte: Dados da pesquisa.

Além disso, uma condição necessária para que um projeto de investimento seja economicamente viável é que o B/C gerado seja maior que a unidade. Assim, de acordo com a Tabela 6 e com a Figura 2, constatou-se que, no Estado do Rio Grande do Sul, há $45 \%$ de probabilidade de o B/C ser menor que a unidade, ou seja, de não gerar um benefício líquido econômico maior que o custo de investimento, e $55 \%$ de probabilidade de o B/C ser maior que a unidade e a atividade ser economicamente viável. Já no Estado do Paraná, há $40 \%$ de probabilidade de o B/C ser menor que a unidade e $60 \%$ de ele ser maior. 


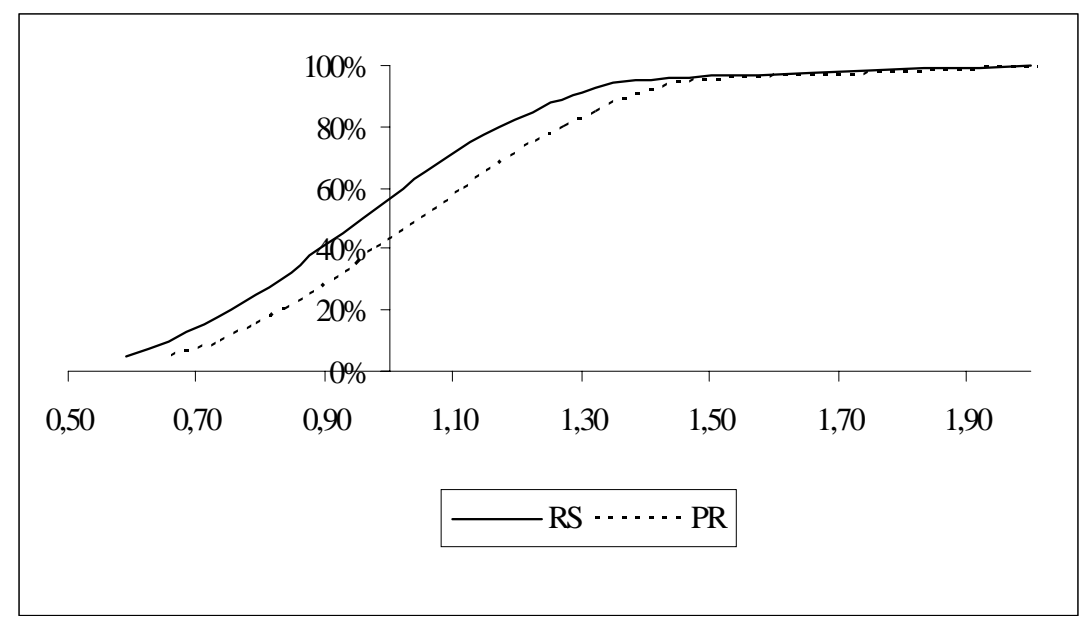

Figura 2 - Análise de risco no cultivo de trigo nos Estados do Rio Grande do Sul e Paraná, pela distribuição acumulada da probabilidade de ocorrência do B/C.

Fonte: Dados da pesquisa.

Assim como na análise sem risco, evidenciou-se que a produção de trigo é mais rentável economicamente no Paraná, mesmo levando em consideração os riscos inerentes às oscilações nos preços do grão, na produtividade, nos custos com colheita mecânica, uréia, fertilizante, sementes, fungicida e na taxa de juros. Isso se deve especialmente ao fato de o nível de preço e de produtividade ter sido maior, em média, no Estado do Paraná, como pode ser visto nas Tabelas 1, 2 e 3 (Anexos), apesar da oscilação dessas variáveis.

\section{Conclusões}

De acordo com os resultados, o investimento na produção de trigo em ambos os Estados, Rio Grande do Sul e Paraná, é economicamente viável, principalmente nesta última região, que possui maior nível médio de preço do grão e maior nível de produtividade. Em ambas os estados, o coeficiente obtido para o indicador $\mathrm{B} / \mathrm{C}$ foi maior que a unidade; $\mathrm{o}$ VPL 
foi positivo; e a TIR e TIRM foram maiores que a taxa de juros de $0,59 \%$ ao mês, o que indica que a triticultura remunera e incrementa o capital investido.

No entanto, sob condições de risco, evidencia-se a fragilidade do cultivo do trigo em gerar benefícios econômicos líquidos tanto no Rio Grande do Sul como no Paraná, e há $45 \%$ e $40 \%$ de probabilidade de a produção resultar em prejuízos, de acordo com o indicador $\mathrm{B} / \mathrm{C}$ simulado pelo método de Monte Carlo, no Rio Grande do Sul e no Paraná, respectivamente, razão pela qual a cultura é arriscada.

A análise de sensibilidade realizada evidenciou que a maior parcela do risco se deve à variação no nível de preço e de produtividade do trigo, o que mostra a importância das políticas de garantia de preço e de investimento para o melhoramento genético da cultura. Assim, reconhecem-se como relevantes a análise de preço como instrumento de planejamento da atividade e a aquisição de cultivares que resultem no maior nível de produtividade do trigo.

Em síntese, pode-se concluir que a triticultura é economicamente viável em ambos os estados, Rio Grande do Sul e Paraná, e pode ser utilizada economicamente como uma cultura de inverno e como alternativa agronômica para rotação da terra e manutenção do controle de doenças como o nematóide cisto da soja, uma vez que o trigo é uma cultura resistente a esse hospedeiro.

\title{
Referências
}

\begin{abstract}
ANUÁRIO DA AGRICULTURA BRASILEIRA (AGRIANUAL). CONSULTORIA E AGROINFORMSTIVOS (FNP). São Paulo: 2008. 502 p.
\end{abstract}

BUARQUE, C. Avaliação econômica de projetos: uma apresentação didática. 8. ed. Rio de Janeiro: Campus, 1991. 266 p. 
Matheus Wemerson Gomes Pereira,

Alan Figueiredo de Arêdes \& Erly Cardoso Teixeira

COMPANHIA NACIONAL DE ABASTECIMENTO (CONAB). Central de informações agropecuárias..Disponívelvem: $<\mathrm{http}: / /$ w w w. c on ab.gov.br/con abweb/download/s a fra/ 2_levantamento_200708.pdfc> Acesso em: 22 fev. 2008.

CONFEDERAÇÃO NACIONAL DA AGRICULTURA ABASTECIMENTO (de Portugal) (CNA). Disponível em:< http:// www.cna.pt/> Acesso em: 05 set. 2007.

Empresa Brasileira de Pesquisa Agropecuária (EMBRAPA). Tecnologia de produção de soja para a região central do Brasil. Londrina: Embrapa, Embrapa Agropecuária Oeste, 228 p. 2007.

Empresa Brasileira de Pesquisa Agropecuária (EMBRAPA). Disponível em:< http://www.embrapa.br/> Acesso em: 05 set. 2007.

GITMAN, L. J. Princípios de administração financeira. 7. ed. São Paulo: Harbra, 1997.

NORONHA, J. F. Projetos Agropecuários: administração financeira, orçamento e viabilidade econômica. 2. ed. São Paulo: Editora S. A, 1988. 269 p.

REZENDE, J. L. P.; OLIVEIRA, A. D. Análise econômica e social de projetos florestais. Viçosa: Editora UFV, 2001. 389 p.

ROSS, S. A.; WESTERFIELD, R. W.; JORDAN, B. D. Princípios de administração financeira. 2. ed. São Paulo: Atlas, 1998. 425 p.

WOILER, S.; MATHIAS, W. F. Projetos: planejamento, elaboração e análise. São Paulo: Atlas, 1996. 294 p. 
Abstract - The objective of this paper is to analyze the economic viability of wheat production in the states of Rio Grande do Sul (RS) and Paraná (PR), with and without risk conditions. The applied methodology includes the indicators of economic viability Benefit-Cost, Net Present Value, Internal Rate of Return, Internal Rate of Return Modified and the application Monte Carlo method. The results suggest the wheat production is economically viable in the state of Paraná.

Key-words: Rio Grande do Sul, Paraná, wheat, economic viability. 
Matheus Wemerson Gomes Pereira,

Alan Figueiredo de Arêdes \& Erly Cardoso Teixeira

\section{Anexo 1}

Tabela 1: Preços reais do quilo do trigo ${ }^{1}$ recebido pelos produtores, 1998 a 2007

\begin{tabular}{ccc}
\hline \multirow{2}{*}{ Ano } & \multicolumn{2}{c}{ Estado } \\
\cline { 2 - 3 } & RS & PR \\
\hline 1998 & 21,10 & 21,93 \\
1999 & 25,18 & 25,70 \\
2000 & 23,51 & 24,80 \\
2001 & 25,46 & 26,16 \\
2002 & 32,73 & 34,28 \\
2003 & 33,06 & 33,67 \\
2004 & 25,94 & 28,82 \\
2005 & 19,35 & 21,16 \\
2006 & 20,71 & 22,60 \\
2007 & 24,03 & 26,23 \\
\hline Média & 25,11 & 26,54 \\
Desvio-padrão & 4,65 & 4,54 \\
Coeficiente de variação & 18,51 & 17,12 \\
\hline
\end{tabular}

${ }^{1}$ Deflacionado pelo IGP-DI.

Fonte: Agrianual (2008) 
Tabela 2: Fluxo de caixa elaborado para o cultivo de trigo no Estado do Rio Grande do Sul (valores em R \$)

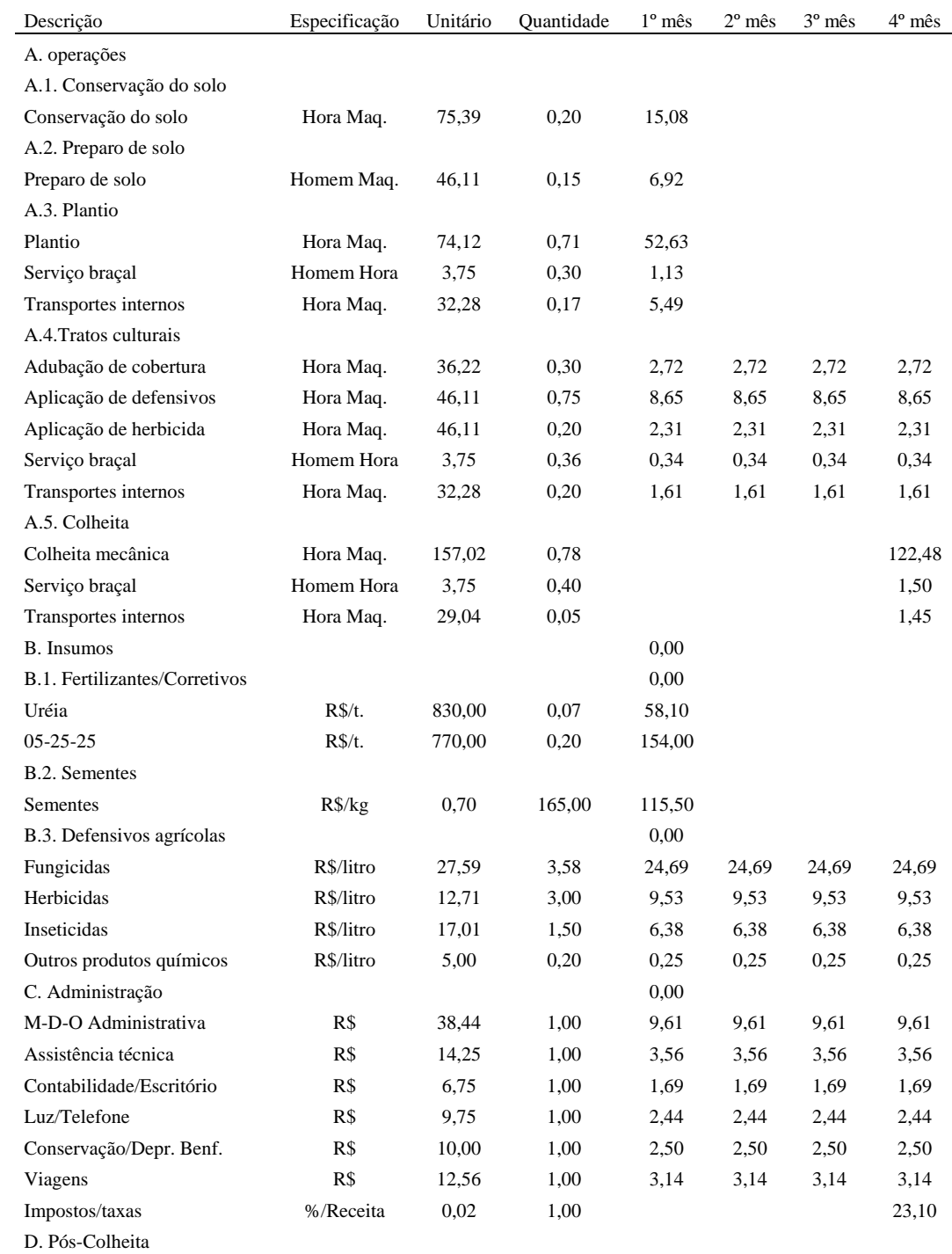


Matheus Wemerson Gomes Pereira,

Alan Figueiredo de Arêdes \& Erly Cardoso Teixeira

\begin{tabular}{|c|c|c|c|c|c|c|c|}
\hline Transportes internos & $\mathrm{R} \$$ & 10,40 & 2,40 & & & & 24,96 \\
\hline Recepção, secagem e limpeza & $\mathrm{R} \$$ & 17,00 & 2,40 & & & & 40,80 \\
\hline Armazém (1 mês) & $\mathrm{R} \$$ & 3,33 & 2,40 & & & & 7,99 \\
\hline Taxa administrativa & $\mathrm{R} \$$ & 2,03 & 2,40 & & & & 4,87 \\
\hline Custo total & $\mathrm{R} \$ / \mathrm{ha}$ & & & 488,24 & 79,41 & 79,41 & 306,56 \\
\hline Preço trigo & $\mathrm{Sc}$ & & & & & & 25,11 \\
\hline Produção & Sc/ha & & & & & & 40,00 \\
\hline Receita & $\mathrm{R} \$ / \mathrm{ha}$ & & & & & & 1004,28 \\
\hline Fluxo líquido de caixa & $\mathrm{R} \$ / \mathrm{ha}$ & & & $-488,24$ & $-79,41$ & $-79,41$ & 697,72 \\
\hline
\end{tabular}

Fonte: Elaborado pelos autores.

Tabela 3: Fluxo de caixa elaborado para o cultivo de trigo no Estado do Paraná (valores em R\$)

\begin{tabular}{|c|c|c|c|c|c|c|c|}
\hline Descrição & Especificação & $\begin{array}{c}\text { Valor } \\
\text { Unitário }\end{array}$ & Quantidade & $1^{\circ}$ mês & $2^{\circ}$ mês & $3^{\circ}$ mês & $4^{\circ}$ mês \\
\hline \multicolumn{8}{|l|}{ A. operações } \\
\hline \multicolumn{8}{|l|}{ A.1. Conservação do solo } \\
\hline Conservação do solo & Hora Maq. & 75,39 & 0,20 & 15,08 & & & \\
\hline \multicolumn{8}{|l|}{ A.2. Preparo de solo } \\
\hline Preparo de solo & Hora Maq. & 46,11 & 0,15 & 6,92 & & & \\
\hline \multicolumn{8}{|l|}{ A.3. Plantio } \\
\hline Plantio & Hora Maq. & 74,12 & 0,60 & 44,47 & & & \\
\hline Serviço braçal & Homem Hora & 3,75 & 0,30 & 1,13 & & & \\
\hline Transportes internos & Hora Maq. & 32,28 & 0,25 & 8,07 & & & \\
\hline \multicolumn{8}{|l|}{ A.4.Tratos culturais } \\
\hline Adubação de cobertura & Hora Maq. & 36,22 & 0,30 & 2,72 & 2,72 & 2,72 & 2,72 \\
\hline Aplicação de defensivos & Hora Maq. & 46,11 & 0,75 & 8,65 & 8,65 & 8,65 & 8,65 \\
\hline Aplicação de herbicida & Hora Maq. & 46,11 & 0,20 & 2,31 & 2,31 & 2,31 & 2,31 \\
\hline Serviço braçal & Homem Hora & 3,75 & 0,39 & 0,37 & 0,37 & 0,37 & 0,37 \\
\hline Transportes internos & Hora Maq. & 32,28 & 0,40 & 3,23 & 3,23 & 3,23 & 3,23 \\
\hline \multicolumn{8}{|l|}{ A.5. Colheita } \\
\hline Colheita mecânica & Hora Maq. & 157,02 & 0,66 & & & & 103,63 \\
\hline Serviço braçal & Homem Hora & 3,75 & 0,30 & & & & 1,13 \\
\hline Transportes internos & Hora Maq. & 29,04 & 0,05 & & & & 1,45 \\
\hline B. Insumos & & & & 0,00 & & & \\
\hline B.1. Fertilizantes/Corretivos & & & & 0,00 & & & \\
\hline Uréia & $\mathrm{R} \$ / \mathrm{t}$. & 882,47 & 0,09 & 79,42 & & & \\
\hline $05-25-25$ & $\mathrm{R} \$ / \mathrm{t}$. & 746,32 & 0,20 & 149,26 & & & \\
\hline \multicolumn{8}{|l|}{ B.2. Sementes } \\
\hline Sementes & $\mathrm{R} \$ / \mathrm{kg}$ & 0,98 & 146,00 & 143,08 & & & \\
\hline B.3. Defensivos agrícolas & & & & 0,00 & & & \\
\hline Fungicidas & $\mathrm{R} \$$ /litro & 79,32 & 1,32 & 26,18 & 26,18 & 26,18 & 26,18 \\
\hline Herbicidas & $\mathrm{R} \$ /$ litro & 6,28 & 5,60 & 8,79 & 8,79 & 8,79 & 8,79 \\
\hline
\end{tabular}


REVISTA DE ECONOMIA E AGRONEGÓCIO, VOL.5, $N^{o} 4$

\begin{tabular}{|c|c|c|c|c|c|c|c|}
\hline Inseticidas & $\mathrm{R} \$ /$ litro & 391,85 & 0,10 & 9,80 & 9,80 & 9,80 & 9,80 \\
\hline Outros produtos químicos & $\mathrm{R} \$ /$ litro & 6,90 & 0,20 & 0,35 & 0,35 & 0,35 & 0,35 \\
\hline C. Administração & & & & 0,00 & & & \\
\hline M-D-O Administrativa & $\mathrm{R} \$$ & 38,44 & 1,00 & 9,61 & 9,61 & 9,61 & 9,61 \\
\hline Assistência técnica & $\mathrm{R} \$$ & 14,25 & 1,00 & 3,56 & 3,56 & 3,56 & 3,56 \\
\hline Contabilidade/Escritório & $\mathrm{R} \$$ & 6,75 & 1,00 & 1,69 & 1,69 & 1,69 & 1,69 \\
\hline Luz/Telefone & $\mathrm{R} \$$ & 9,75 & 1,00 & 2,44 & 2,44 & 2,44 & 2,44 \\
\hline Conservação/Depr. Benf. & $\mathrm{R} \$$ & 10,00 & 1,00 & 2,50 & 2,50 & 2,50 & 2,50 \\
\hline Viagens & $\mathrm{R} \$$ & 12,56 & 1,00 & 3,14 & 3,14 & 3,14 & 3,14 \\
\hline Impostos/taxas & $\% /$ Receita & 0,02 & 1,00 & & & & 27,46 \\
\hline \multicolumn{8}{|l|}{ D. Pós-Colheita } \\
\hline Transportes internos & $\mathrm{R} \$$ & 16,15 & 2,70 & & & & 43,61 \\
\hline Recepção, secagem e limpeza & $\mathrm{R} \$$ & 20,83 & 2,70 & & & & 56,24 \\
\hline Armazém (1 mês) & $\mathrm{R} \$$ & 3,33 & 2,70 & & & & 8,99 \\
\hline Taxa administrativa & $\mathrm{R} \$$ & 2,42 & 2,70 & & & & 6,53 \\
\hline Custo total & $\mathrm{R} \$ / \mathrm{ha}$ & & & 532,74 & 85,31 & 85,31 & 334,35 \\
\hline Preço trigo & $\mathrm{Sc}$ & & & & & & 26,54 \\
\hline Produção & Sc/ha & & & & & & 45,00 \\
\hline Receita & $\mathrm{R} \$ / \mathrm{ha}$ & & & & & & 1194,08 \\
\hline Fluxo líquido de caixa & $\mathrm{R} \$ / \mathrm{ha}$ & & & $-532,74$ & $-85,31$ & $-85,31$ & 859,72 \\
\hline
\end{tabular}

Fonte: Elaborado pelos autores. 